\title{
J Clifton Edgar (1859-1939) of New York and his obstetric text
}

\section{P M Dunn}

Arch Dis Child Fetal Neonatal Ed 2005;90:F441-F443. doi: 10.1136/adc.2004.063503

Edgar, a leading academic obstetrician/gynaecologist in the United States at the beginning of the 20th century, explored a number of interesting aspects of pregnancy and childbirth in his 1903 textbook on obstetrics.

Correspondence to: Professor Dunn

Department of Child Health, University of Bristol, Southmead Hospital, Southmead, Bristol BS10 5BN, UK; P.M.Dunn@bristol.ac.uk

Accepted 23 August 2004 $\int$ A ames Clifton Edgar, the son of James Alexander and Mary Eliza Coe Edgar, was born in New York on 14 June 1859. He attended St Paul's School at Concord, New Hampshire, and Lafayette College from which he received the degree of $\mathrm{PhB}$ in 1882 and MA in 1884. In 1884 he graduated MD from New York Medical College standing first in a class of 180 students. During 1885-1887, Edgar served as an interne, assistant curator, and attending physician specialising in diseases of women at Bellvue Hospital, New York. This was followed in 18871888 by the post of instructor in pathology at New York University. Next he spent a year abroad undertaking postgraduate studies at the Royal Frauenklinik in Munich and at the Prague Maternity Hospital. On returning he lectured on obstetrics at New York University, being appointed adjunct professor (1889), associate professor (1892), and full professor in 1898 (fig 1). Two years later in 1990, he was appointed professor of obstetrics and clinical midwifery in Cornell University Medical College, a post he professor. During these years he built up a large private practice and served on the staff of a number of hospitals including the Bellvue LyingIn, the Mother's and Babies Hospital, Manhattan Maternity, the New York Maternity, the Jewish Maternity, and the Greenwich Hospital. He retired from medical practice in 1932.

Edgar was a fellow of the American College of Surgeons and of the New York Academy of Medicine. Among the many other societies of which he was a member were the American Gynecological Society, the American Medical Association, and the New York Obstetrical Society. He was president of the latter in 1908. He was also a corresponding fellow of the Munich Gynecological Society.

The end of the 19th century saw great changes in practice as obstetrics developed from being a medical speciality to becoming more a surgical one. Edgar was intensely interested in these changes and took an active interest in all advances in scientific obstetrics. Although his teaching methods were delightfully informal, he impressed his audience by his ability to identify and emphasise facts that were really important. held until 1926 when he became emeritus
He was greatly respected and indeed revered by his students.

Edgar was the author of a number of articles in medical journals and books. Easily his most important publication was his textbook The practice of obstetrics, first published in 1903 and running through five editions. The extracts that follow are taken from the 1 st edition. ${ }^{1}$ They give a flavour of his lively and interesting approach to the subject.

\section{On drug abuse during pregnancy ${ }^{1}$}

"Alcoholism: It was not until 1900 that Nicloux was able to demonstrate that the drug in question enters the fetal circulation. He gave a woman milk-punches after she was well along toward delivery, and was able to distil some of the alcohol from the blood of the umbilical vein. In regard to the effects of alcoholism on pregnancy, as studied in a series of chronic drunkards, there is no evidence of any strong tendency to abortion. Still-birth is quite common, but the principal effect of alcohol upon the fetus is shown in the extraordinary tendency to

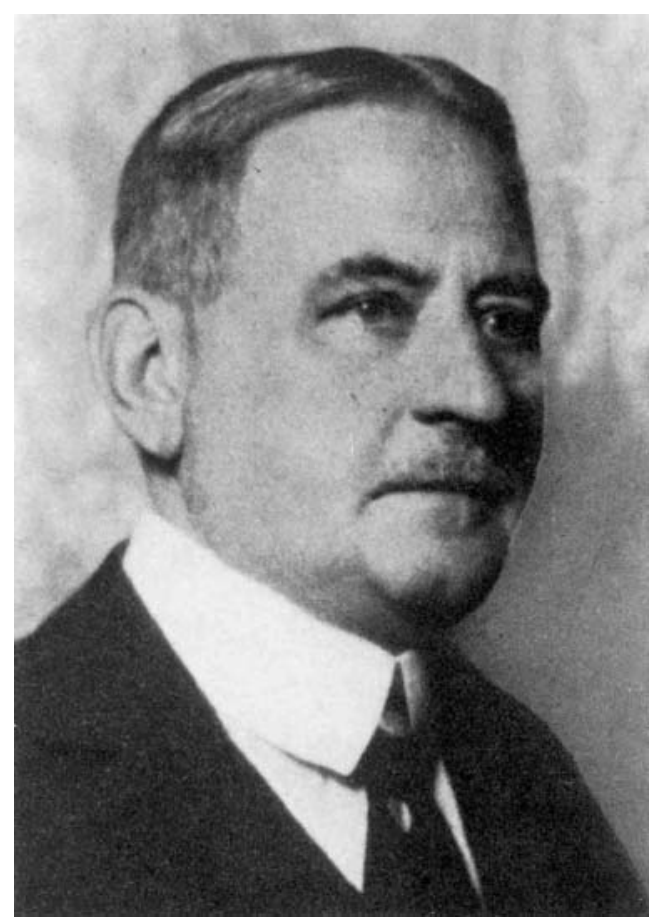

Figure 1 James Clifton Edgar (1859-1939). 
nervous and cerebral disease, malformation, and degeneracy exhibited by these children as they grow up. Alcohol in the fetal circulation tends to arrest the highest development.

Morphinism: In pregnant morphinomaniacs no especial tendency to abortion or premature delivery has been noted. The children born to such women often appear healthy in every respect. Morphin, however, has been recovered from the fetal tissues. There is considerable evidence that the fetuses are more likely than others to require resuscitation at birth. I have observed that attempts at withdrawal of the morphin during gestation have been followed by excessive fetal movements, which again subsided when the use of the drug was renewed. This phenomenon suggest that the fetus has acquired a tolerance to the narcotic.

Nicotinism: The pregnancies in many tobacco factories, etc., are not interrupted, although there is a very high mortality among the children of these women. Nicotinism tends to the production of weak, undersized fetuses with a high degree of secondary mortality."

Edgar was extremely interested in the shape of the folded fetus in utero, in its presentation (fig 2), and in the influence of the mother's posture during labour. In the extracts below he describes the effect of moulding of the fetal skull during labour and the method of artificially rupturing the membranes.

\section{On fetal moulding'}

"The result of the pressure of the birth canal upon the fetal skull is to diminish the capacity of the whole cranium. This is brought about by: (1) The approximation and overlapping of the bones of the vertex. The bones of the calvarium are not merely joined by membrane but there is considerable opportunity for overlapping under pressure, since (a) they ossify late; (b) they are separated by sutures and fontanelles which permit of overlapping; (c) and they are so thin as to admit of bending and moulding. Overlapping in the process of labour always takes place in a systematic manner. The parietal bones overlap the frontal and the occipital bones, and the parietal bone which is submitted to the greater pressure - that is, always the one which lies posterior in the pelvis - slides under its fellow. (2) The cerebrospinal fluid is squeezed out of the head into the spinal canal. (3) The blood is also forced out of the cerebral vessels, to a certain extent. (4) Then, too, the brain substance itself in the fetus is but slightly developed, and is therefore capable of being compressed and moulded to a considerable degree without any permanent damage to the fetus."

\section{On artificial rupture of the membranes'}

"The membranes are perforated by a uterine sound or similar instrument passed in and through the os under the guidance of the left index-finger in the vagina, and the liquor amnii is allowed to drain away. It is chiefly useful as an adjuvant to other methods. It should not be used after the fetus is viable, since the fetus is exposed to prolonged pressure and the mother to the dangers of 'dry labor'. There are some exceptions to this rule, however; e.g. accidental hemorrhage, hydramnios. This procedure is frequently employed with criminal intentions."
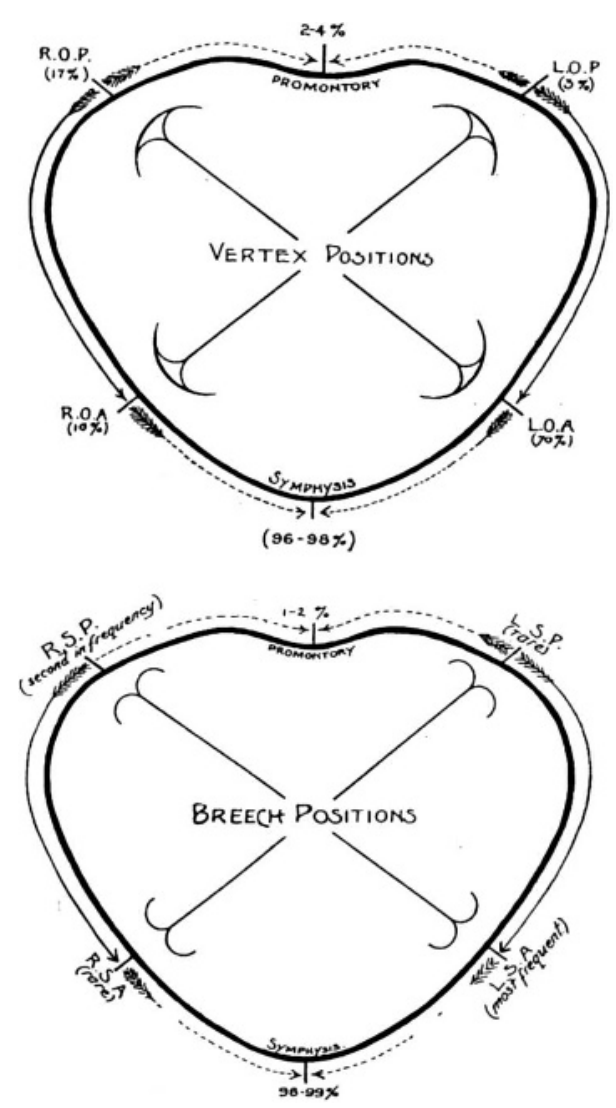

Figure 2 Frequency of fetal presentation with vertex and breech positions.'

Edgar wrote at length on the management of the infant at birth. On asphyxia neonatorum, which he divided into the blue and white forms, he wrote:

"This subject, at first sight one of great simplicity, is in reality one of the most difficult in the entire subject of obstetrics. The simple and straightforward manner in which it is presented by the great majority of authors involves a discreet suppression of numerous problems which would otherwise confuse and perplex the student ... Asphyxia neonatorum is one of the neglected connecting links between the two specialties - obstetrics and pediatrics."

\section{On resuscitation and ligation of the cord ${ }^{1}$}

"Respiration being fully established, the ligation of the cord should be delayed until pulsations cease, unless there is some positive indication to the contrary. Immediate ligation deprives the fetus of about three ounces of blood ... If, after the expulsion of the child, cyanosis is evident, the cord should be divided at once and allowed to bleed about an ounce. It should be remembered, however, that immediate ligation of the cord is in itself equivalent to depriving the child of an ounce of blood. In the anemic form of asphyxia we should cover the child with hot flannels and wait for the pulsations of the cord to cease ... The second indication is to cleanse the respiratory passages of fluids aspirated during labor ... after the preliminaries just described either attempt to excite natural 


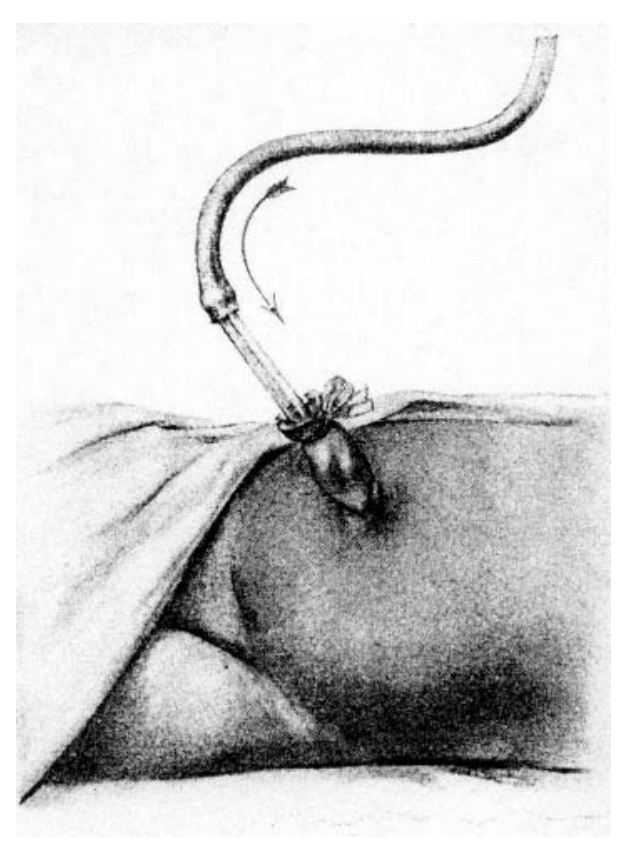

Figure 3 Umbilical cannula for the infusion of fructosate of soda in the treatment of severe birth asphyxia.'

respiration by reflex stimuli, or in more serious cases proceed at once to artificial respiration ... All attempts to enter the larynx should be frowned upon. Practice upon the cadaver will readily impress one with the barbarousness of such an attempt upon the newly born child."

\section{On umbilical infusion of fructosate of soda for severe birth asphyxia'}

"Success has attended infusion through the umbilical vein after all the customary resources have proved inefficient. The infusion is something more than an ordinary saline solution, for Schücking (1902) added fructosate of soda, a substance which is believed to take up the excess of carbonic acid in the blood with the formation of sodium carbonate, and sugar. The formula used is as follows: fructosate of soda, 0.5; sodium chloride, 0.7; boiled water, $50 \mathrm{cc}$. The apparatus employed is a graduated bottle, a tube, and a cannula (fig 3). The umbilical vein is cut across and the cannula inserted. Thirty cubic centimetres are thrown in at first, followed by a second infusion of $20 \mathrm{cc}$. As soon as the heart and respiration start up, ordinary measures are resumed."
In the 1890s caesarean section had emerged as a relatively safe procedure owing to anaesthesia and aseptic surgery, especially in the management of disproportion. Edgar's comments on its use at that time are of great interest.

\section{On indications for caesarean section'}

"The indications for this operation are of two kinds positive and relative. Caesarean section is positively indicated when the maternal or fetal dystocia is so great that it is impossible to remove the fetus even after embryotomy. The relative indications for the operation are not so clearly marked. When it is evident that embryotomy can be done successfully and without great risk to the mother, the question in the presence of a dead child is easily decided, but if the child is alive the proper course is not so clear. The good results which have recently followed Caesarean section have led many operators to consider, that a conjugative of 3 inches $(7.62 \mathrm{~cm})$ with the child living, and 2.5 inches $(6.35 \mathrm{~cm})$ with the child dead, requires the operation. It is to be remembered that in cases in which the difficulty is due to a flat rather than a generally contracted pelvis, a shorter conjugate will suffice to effect delivery through the natural passages ... As a rule, Caesarean section should be done at term, but it is not necessary to wait for labor to begin. A point in favour of the Caesarean operation is that by it measures can be taken to prevent future conceptions by tying and dividing the Fallopian tubes. While pelvic deformity is the commonest condition which requires this mode of delivery, pelvic tumours of almost any kind may be the cause of the dystocia. Eclampsia and placenta praevia have sometimes been put down as conditions which may occasionally demand Caesarean section ...

When the environment is favourable, when conveniences and competent assistants are at hand, when the mother is in good condition and has not been infected by repeated examinations and unsuccessful attempts at delivery, and when the fetus is still strong and healthy in the uterus, the danger of Caesarean section to the mother is almost nil, and we can assure the patient and her family that the child will almost certainly survive."

Edgar died at the age of 79 on 7 April 1939 at his home in Round Hill Road, Greenwich, Connecticut. He was survived by his wife Ellen Muriel Beatrice Soutter and his two sons, Clifton and Charles.

\section{REFERENCE}

1 Edgar JC. The practice of obstetrics. London: Rebman Ltd, 1903. 